\title{
Münsteraner Rechtsanwalt Dr. Peter Wigge wurde zum Honorarprofessor an der Westfälischen Wilhelms-Universität Münster ernannt
}

Münster, 12.06.2014. Die Rechtswissenschaftliche Fakultät der Westfälischen Wilhelms-Universität Münster hat Herrn Rechtsanwalt Dr. Peter Wigge in den Kreis ihrer Honorarprofessoren aufgenommen. Mit der Ernennung zum Honorarprofessor würdigt die Rechtswissenschaftliche Fakultät die Verdienste von Herrn Dr. Wigge in Lehre und Forschung auf dem Gebiet des Medizin- und Gesundheitsrechts. Herr Dr. Wigge wirkt dort seit langem mit großem Einsatz und herausragendem Erfolg als Lehrbeauftragter der Fakultät. Als bundesweit anerkannter Fachmann verbindet er in besonderer Weise die Fragestellungen der Praxis - so- wohl der anwaltlichen Tätigkeit als auch der Gesetzgebung sowie der (Selbst-) Verwaltung der gesetzlichen Krankenversicherung - mit der rechtswissenschaftlichen Analyse.

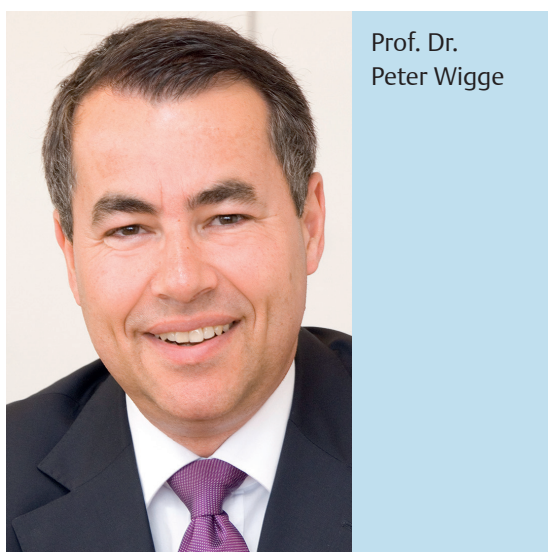

Prof. Dr. Wigge ist Justiziar der Deutschen Röntgengesellschaft (DRG), der wissenschaftlichen Gesellschaft für medizinische Radiologie. Er berät die DRG insbesondere in fachspezifischen Fragestellungen wie der Röntgenverordnung, dem Aus- und Weiterbildungsrecht und der Zusammenarbeit mit anderen Fachgebieten. 